\title{
LITE: THE LARGE IMAGING TELESCOPE
}

\author{
L. VIGROUX \\ DAPNIA \\ Service d'Astrophysique \\ CEN Saclay \\ France
}

It was realised very early in the development of the ESO 16 metre equivalent Very Large Telescope (VLT) that wide-field imaging is too complicated and costly to implement on the VLT itself and should be done with a smaller telescope.

Accompanying imaging observations are essential for the optimal use of the VLT. Let's take an example. For large-scale structure studies, the VLT allows the measurement of redshifts in a 30 arcmin field-of-view of galaxies of magnitude 23 or even fainter. They are too faint to be reliably detected on Schmidt plates, so the input observation catalogue must be obtained from deep CCD imaging. In this example, outstanding image quality is needed to make a clear separation between faint galaxies and stars. The VLT will have in its imaging mode a 7 arcmin field and is not usable for obtaining such images. The best compromise is a middle-size telescope of about $2.5 \mathrm{~m}$ diameter and equipped with a wide-field CCD camera.

These considerations have pushed to the emergence of a new project of a Large Field Imaging Telescope (LITE) proposed by a French and German consortium. French laboratories include Observatoire de Meudon, Institut d'Astrophysique de Paris, Observatoire Midi Pyrénnées, Observatoire de Besançon, Observatoire de Marseille, and are led by the Department of Astrophysics and Particle Physics in Saclay. The German group include Sonneberg Observatory, Tautenburg Observatory and the Institute of Astrophysics in Potsdam.

While this project was originally designed for observations of mainly cosmological interest, it has the technical capabilities to cover a much broader range of astrophysical problems. The consortium is now working on several programmes.

Galactic structure study will take advantage of the deep images obtained for extragalactic purposes, with the addition of the observations of selected galactic fields, in particular in the thick disk region. The main emphasis is in the study of the low mass star luminosity function. On a 10 year time scale, we also can detect the proper motions of a large number of faint stars and we expect that the combined information will improve our knowledge of the structure and evolution of the Galaxy. These fields will be monitored to detect and analyze the variable stars, and to obtain a catalogue fainter than was already existing. A by-product of this analysis will be a large scale extinction map, and the study of Galactic Cirrus at a scale smaller than the IRAS resolution.

Programmes on nearby galaxies will include the determination of colour gradients of early type galaxies, the analysis of which is now limited by the poor statistics of the available samples. The LITE survey will be an invaluable tool to study the relationship between the environment and the galaxy morphology and luminosity distribution, a related topic being the search for very low 
surface brightness galaxies. These domains are still open. For example our present knowledge of the morphology density relation is based on the Dressler sample that contains only 6000 galaxies. With LITE, we may be able to study this relation on a sample larger by a factor of at least 10. A systematic search for starburst galaxies is also planned using slitless low resolution spectroscopy. It will allow us to investigate their spatial distribution, their luminosity function, their evolutionary stage, and the significance of triggering mechanisms. This survey will also be a new opportunity for examining the existence of primordial galaxies at low redshift.

LITE will offer an efficient way of mapping the large-scale galaxy distribution out to redshifts of 0.6-0.8. The existing faint catalogues over significant areas of the sky were obtained from digitisation of photographic plates, yielding a limiting magnitude of $m_{B} \sim 22$. However, the nonlinearity and coarse spatial resolution of the photographic emulsion call into question the reliability of galaxy catalogues at the plate limit. LITE will provide the necessary galaxy catalogue for performing deep redshift surveys to $m_{B} \sim 22-23$ over several tens of square degrees of the sky using multi-object VLT instruments. These redshift surveys are needed for understanding the nature of the intercepted over- and under-densities of galaxies in the existing narrow pencil-beam probes, and for putting reliable limits on the typical and largest size for the large-scale structures. If a faint object spectrograph with a multiplex capabilities larger than 100 objects by exposure is available at the VLT, such a programme would require about 100 nights of observations, well within the possibilities of a VLT key programme.

From the galaxy catalogue, LITE will be able to detect clusters of galaxies by correlation analysis up to redshifts of 1.0. Coupled with redshift measurements on the VLT, the cluster catalogue will provide a unique sample for studying cluster dynamics, their evolutionary stages, their content in dark matter, and their relationship with the large-scale structures. Moreover, LITE will be in operation at the same time as the new generation of X-ray satellites, AXAF and XMM. We can therefore foresee large coordinated programmes on distant clusters.

From the large galaxy catalogue, we also plan an unprecedented search for gravitational distortions by dark matter large-scale structures. In analogy with the gravitational lensing effect by dark matter halos in clusters of galaxies, dark matter distributed over larger scales would lead to a significant number of distorted images of faint background galaxies. A statistical detection of alignments of faint galaxies could be obtained in spite of their intrinsic morphological variations thanks to the large number of objects in the sample. Given the performances of LITE, the detection of large structures of dark matter will be optimal in the redshift range 0.2-0.4 using source galaxies in the redshift range 0.5-0.8.

A very deep photometric survey to $m_{B} \sim 26$ is also planned for deriving new constraints on galaxy evolution over cosmological time scales. Multicolour counts of objects per magnitude interval in a catalogue more than an order of magnitude larger than the existing samples will put new constraints on the merging processes recently suggested as a major clue to recent galaxy evolution. The excellent resolution of the images obtained with LITE will provide direct estimates of the rate of occurrence of close pairs and merging systems and its dependence on redshift. Along these lines, the galaxy redshift survey at brighter magnitudes will put constraints on the influence of the environment on the evolution of galaxies, and will allow the measurement of the slope of the galaxy luminosity at the faint end, a crucial parameter in evolutionary models.

Low-resolution spectroscopy of the large number of compact objects detected with LITE will yield a complete and homogeneous catalogue of QSOs. The absence of the biases present in existing catalogues and resulting from the multi-colour selection procedure, will allow a new examination of the QSO luminosity function and the apparent redshift cut-off in their distribution (at 3). The homogeneity of the catalogue will also greatly benefit the understanding of the QSO- 
AGN connection. Using the high quality images we will be able to study the environment of low redshift QSOs and their relationship to the large-scale galaxy distribution. Acquisition of the spectra with the VLT (FUEGOS and/or FORS) for the selected candidates will allow us to probe the distribution and properties of known absorbers along the QSO's line of sight (galaxies surrounded by large envelopes, etc.). New types of absorbers may also be discovered (metal enriched intergalactic clouds for example). The combination of spectroscopy and high quality imaging will provide for the first time a firm basis for a model describing the formation and evolution of halos around galaxies. New cases of lensed QSOs will also be found, and monitoring the variability of the images will put constraints on the value of the Hubble constant.

LITE will be used to detect distant supernovae. In 100 square degrees, we will be able to discover $\mathbf{4 0}$ supernovae up to a redshift of 0.5 each week. The advantage of LITE for this search is of course its very good image quality and photometric performances which will help to detect supernovae as faint as $m_{v}=23$ embedded in distant galaxies. The redshift determination will be done with the VLT.

Another domain will be the continuation of the brown dwarf research by microlensing effects on stars in the Magellanic Clouds. The two existing experiments are based on a $40 \mathrm{~cm}$ telescope and a 4 million pixels CCD camera for the French MACHO instrument at ESO, and a $1.20 \mathrm{~m}$ telescope and a double 4 million pixels CCD camera for the Australian-American instrument at Mount Stromlo. LITE will provide a gain by a factor 10 compared to these instruments. The results from the first experiments are very promising, with the detection of 3 possible microlensing events. Further observations with these experiments will hopefully confirm the existence of the halo brown dwarfs, but their mass distribution must wait for the observations with LITE.

Three types of observational programmes are considered:

1) a multicolour astrometric and photometric survey in individual fields selected according to

Galactic structure and stellar programme;

2) a multicolour and slitless, low resolution spectroscopic survey of typically 100 square degrees

for cosmological observations and supernovae research; and

3) observations in front of the Magellanic Clouds for detection of brown dwarfs.

All of these programmes require very good image quality. Imaging will be done from the UV to the near infrared.

The scientific requirements call for LITE being a telescope of $2.5 \mathrm{~m}$ diameter with a mean image quality, including seeing, of 0.8 arcsec (or better) over a field of 1.5 degrees (or more). This can only be achieved with good sampling of the image PSF by the CCDs. For a typical pixel pitch of 15 microns, 0.25 arcsec pixels are achieved with $15 \mathrm{~m}$ focal length; this corresponds to an $\mathrm{f} / 5$ aperture ratio. The first design was a quasi Ritchey-Chretien system with a Gascoigne corrector, analog to the Sloan Digital Sky Survey Telescope. A new optical concept worked out at the Tautenburg Observatory, with the assistance of Ray Wilson from ESO is now preferred. It is a modified version of the 3 mirror Paul-Baker telescope which provides a plane focal surface at the 'prime focus' location, behind the secondary mirror. A preliminary design study (U. Laux, this conference) has shown that for a telescope with $2.5 \mathrm{~m}$ diameter and focal ratio $\mathrm{f} / 4$, image quality of $0.4 \mathrm{arcsec}$ can be obtained at the edge of a 2.5 degree field, and significantly better towards the centre. Compared to the initial Cassegrain solution, this design has two important advantages, the absence of chromatic aberrations because there are only reflecting mirrors, and a very easy baffling system to suppress straylight. The first point is the most important, since this 3 mirror telescope is the first design which provides good image quality and throughput from the UV to the near IR. Gascoigne correctors are limited in wavelength 
range due to chromatism of the lenses. A preliminary design of the telescope, following the optical design of $U$. Laux have been done by Carl Zeiss Jena (this conference).

As a baseline, the CCD camera will be organised around thin, back side illuminated Thomson CCDs, each with $2048 \times 2048$ pixels and 15 micron length. These CCDs are being developed for the VLT, and the thick version should become available in 1993 and the thin one in 1994. The three-side buttability provides strips of 2 CCDs width. A 1 square degree surface can be covered with 42 CCDs. Readout time of the whole array will be as low as $\mathbf{3 0}$ seconds, thanks to a parallel acquisition system. Cryogenic temperatures will be provided by a closed cycle cooler in order to simplify the operations. An IR camera will be designed to hold a $3 \times 3$ mosaic of $1024 \times 1024$ pixels arrays, either in InSb (Santa Barbara) or HgCdTe (Rockwell) detectors. Both cameras share the same rotating mechanical support which can place one or the other at the focal plane. The camera bonnette includes filters, grisms and a shutter.

The natural site for this telescope is near the VLT, in the Paranal area, where it may take advantage of the excellent seeing and the large number of photometric nights as compared to the other Chilean sites. Discussions will take place with ESO to study this possibility.

The definition phase of the project will be undertaken in 1993-1994. We must still settle the details of the German-French collaboration, work out the relationships between the consortium and ESO, and obtain funding. The actual start of the project is expected in 1995 and the beginning of the observations in 1999 . In the present status of the project, nothing has been absolutely fixed and new groups are welcome to join.

Projects similar to ours are under development, in particular the Sloan Digital Sky Survey (SDSS) in the U.S.A. We wish to emphasize the differences between our project and the SDSS. The main goal of the SDSS is to make a survey over a large fraction of the entire sky $(\sim \pi$ steradians), both in photometry and in spectroscopy, and with the same telescope. However, the use of the SDSS $2.5 \mathrm{~m}$ telescope for spectroscopic measurements will naturally limit the observations to moderately faint galaxies only. The necessity of the all sky survey pushes towards the largest possible field, but at the detriment of image quality, and to a transit instrument which simplifies the operations.

In our case, the spectroscopic observations are planned with the much larger VLT, which, of course, can reach much deeper. Due to the increasing number of objects at fainter magnitudes, we cannot expect to cover a large fraction of the sky. On the contrary, we shall only be able to obtain images significantly deeper than the SDSS by limiting the sky coverage. For LITE, the priority of optimization is then image quality first, and field-of-view second. In addition, the pointing mode of operation is more suitable for very deep imaging than a transit mode. While many scientific areas are common to both instruments, the trade-offs are different, and the scientific programmes will be different too.

We believe that the combination of the VLT and LITE will offer a unique capability of probing the deep sky and will become a prominent instrument for future cosmological studies. 